\title{
Therapeutic Outcomes of HPV Positive and HPV Negative Oropharyngeal Squamous Cell Carcinomas
}

\section{Aravindh Sivanandan Anand ${ }^{1}$, Gopika Presenavarman ${ }^{1 *}$, Salima Rema Windsor ${ }^{2}$, Priya Venugopaladas Saraswathy ${ }^{3}$}

\author{
${ }^{1}$ Department of Radiotherapy, Govt. Medical College, Trivandrum, India \\ ${ }^{2}$ Department of ENT, Govt. Medical College, Trivandrum, India \\ ${ }^{3}$ Department of Pathology, Govt. Medical College, Trivandrum, India \\ Email: ${ }^{*}$ anandrt2006@yahoo.com
}

How to cite this paper: Anand, A.S., Presenavarman, G., Windsor, S.R. and Saraswathy, P.V. (2020) Therapeutic Outcomes of HPV Positive and HPV Negative Oropharyngeal Squamous Cell Carcinomas. Journal of Cancer Therapy, 11, 507-518. https://doi.org/10.4236/jct.2020.119044

Received: July 17, 2020

Accepted: September 13, 2020

Published: September 16, 2020

Copyright $\odot 2020$ by author(s) and Scientific Research Publishing Inc. This work is licensed under the Creative Commons Attribution International License (CC BY 4.0).

http://creativecommons.org/licenses/by/4.0/

\begin{abstract}
Background \& Objectives: HPV associated (HPV+) oropharyngeal cancers, compared with HPV non-associated ones (HPV-) have different characters and significantly better outcomes. HPV and cancer characteristics differ among countries and few data is available in Indian population regarding this issue. Thus, we attempted to determine the treatment response and survival rates between HPV+ versus HPV negative oropharyngeal cancers in Indian population. Methods: This prospective observational study was conducted from December 2016 - October 2018 in patients with stage III/IV oropharyngeal squamous cell carcinomas $(\mathrm{SCC})(\mathrm{n}=65)$. The patients received induction chemotherapy with Docetaxel, Cisplatin, 5-Fluorouracil $\times 3$ cycles, followed by concurrent chemoradiation 66 Gy as 33 fractions along with cisplatin $40 \mathrm{mg} / \mathrm{m}^{2}$ weekly. The primary outcome was treatment response rate, which was defined as per Response Evaluation Criteria in Solid Tumours (RECIST) criteria. Secondary outcomes were clinico-pathological differences between two groups, overall survival (OS), progression free survival (PFS). Results: Of the 65 patients included in the study, 17 were HPV positive and 48 patients were HPV negative. Median age in HPV positive arm is 48 years and HPV negative arm is 59 years. HPV positive patients presented with early $\mathrm{T}$ stage and advanced nodal (N) stage. Most common histopathology in both arms was moderately differentiated squamous cell carcinomas followed by well differentiated squamous cell carcinomas. HPV+ vs HPV- showed the following, treatment response $82.4 \%$ vs $52.1 \%$ (p-0.029). 1 year progression free survival (PFS) of $76.5 \%$ vs $52.1 \%$ in HPV negative arm (p-0.08) \& 1 year overall survival was $82.4 \%$ vs $70.8 \%$ (p-0.353). Grade 3 or 4 toxicities did not
\end{abstract}


differ significantly between HPV positive and HPV negative arms. Conclusion: HPV positive oropharyngeal SCC patients showed significantly better treatment response than HPV negative ones. Progression free survival, overall survival and toxicity profile did not differ significantly between the two groups. Although due to small size, we did not compare data stratified by the cancer characteristics, the data is worthy to further characterize this tumour especially as HPV positive versus HPV negative.

\section{Keywords}

Oropharyngeal Squamous Cell Carcinoma, HPV, Chemoradiation, Docetaxel, Cisplatin, 5-Fluorouracil, Treatment Response

\section{Introduction}

Head and neck squamous cell carcinoma is the third most common malignancy in both sexes across the globe. It is the most common malignancy in Indian males [1]. Oropharyngeal cancers account for approximately $10 \%$ of the annual worldwide incidence of head and neck squamous cell carcinomas (HNSCC). Overall incidence of HNSCC has fallen in the last three decades; however, the incidence of oropharyngeal carcinoma, mainly tonsil and base of tongue, has been increasing both in United States and Europe.

The incidence of oropharyngeal cancer differs significantly by geography. In United States of America, the annual incidence of oropharyngeal squamous cell carcinoma is $4.8 / 100,000$. The rate has increased by $28 \%$ from 1988 to 2004 largely because of $225 \%$ increase in HPV associated oropharyngeal cancer [2] [3] [4].

The rise in developed countries is unique as other mucosal head and neck cancers have decreased over this same time period. The cause of this is the increasing incidence of HPV associated cancers. The incidence in developing countries is lower at approximately $3 / 100,000$.

The Cancer Atlas project by ICMR (Indian Council of Medical Research) has shown the incidence of various cancers in different parts of India [5]. Kerala has one of the highest incidences of HNSCC which constitutes 30\% of all cancers in India. HPV prevalence in India ranges from $33.6 \%$ in Eastern region to $67 \%$ in South, $15 \%$ in western India.

About 15\% - 20\% of all head and neck squamous cell carcinomas are associated with high risk HPV infection. The highest rates of HPV DNA (upto 70\%) have been found in oropharyngeal squamous cell carcinomas (OSCC) especially the tonsils followed by base of tongue. HPV 16 is the most common type, detected in $90 \%-95 \%$ of HPV related OSCC.

HPV associated oropharyngeal cancers are more likely to occur among men (80\% of whom will not have a smoking history), more common among white individuals, diagnosed in individuals who are 5 to 10 years younger than HPV unassociated oropharyngeal cancers, more common with higher socioeconomic 
status. HPV associated oropharyngeal cancers are characterized frequently as poorly differentiated, non-keratinizing and basaloid in histopathologically [2].

Early stage oropharyngeal cancers are best managed with single modality therapy (radiation or surgery). Treatment of locally advanced tumours involves multiple modalities, the most common being either concomitant chemotherapy and radiotherapy or surgery followed by adjuvant radiotherapy with or without chemotherapy based on pathologic risk factors.

Patients with HPV associated oropharyngeal cancers have significantly better outcomes compared to HPV unassociated oropharyngeal tumours. Novel approaches to surgical and radiation delivery and the incorporation of molecularly targeted chemotherapeutics are currently the focus of clinical investigation with an intent to maximize the therapeutic index for HPV associated oropharyngeal cancers.

There is growing support for the view that the treatment protocol for oropharyngeal cancer should be modified according to HPV status and that de-escalation of treatment intensity may be a real possibility for patients with HPV positive OPSCC.

\section{Relevance of the Study}

High rates of HPV association have been found in oropharyngeal squamous cell carcinomas as per the available literature. Patients with HPV positive oropharyngeal cancers have significantly better survival outcomes compared to HPV unassociated cancers. But the studies comparing the treatment outcomes are sparse from India. Sometimes the rate of incidence of HPV infection in Indian population may be low, hence our study may help to address this issue to some extent.

\section{Aims and Objectives}

\section{Primary Objective}

To assess the treatment response in HPV positive and HPV negative oropharyngeal squamous cell carcinoma

\section{Secondary Objective}

1) Clinicopathological differences between the two arms of study

2) Incidence of HPV positive cases in the study

3) Progression free survival

4) Overall survival

\section{Methodology}

This is a prospective observational study conducted in Department of Radiotherapy, Government Medical College, Trivandrum from Dec 2016 to Oct 2018. Patients with oropharyngeal cancer (all sub sites-Stage III and IV), squamous cell carcinoma (biopsy proven from primary cancer site) and satisfied the inclusion criteria and who expressed willingness were included in the study. Ethical clear- 
ance was obtained prior to the start of the study from Human ethics Committee of the institution.

\section{Inclusion Criteria}

1) Age more than 18 years and less than 70 years of both sexes

2) Biopsy proven (from primary site) cases of squamous cell carcinoma of oropharynx

3) Stage III and IV (locally advanced squamous cell cancers)

4) Eastern cooperative oncology group (ECOG) performance status of 0 to 2

5) Normal blood parameters (CBC, RFT and LFT)

6) Chemotherapy and radiation naive patient

\section{Exclusion Criteria}

1) Patients unwilling for the study and to give written informed consent

2) Clinically significant comorbid conditions

3) Poor performance status of patients (ECOG 3 and 4)

4) Stage I and II

5) Medical contraindication for chemotherapy and radiation

\section{Procedure}

Sixty Five Patients fulfilling the criteria were enrolled in the study. Written informed consent was obtained from each patient prior to the initiation of treatment. Detailed history was elucidated. The master case file was used for data collection. Being a prospective observational study the data of the patients from the start of evaluation to follow up were recorded from the records. These included the following as per the records.

Detailed physical examination including ENT examination (70 degree endoscopy) and biopsy from primary site were taken. Findings will be recorded in the proforma. HPV p16 immunohistochemistry was done in biopsy specimen block. According to this result, patients receiving the standard treatment are grouped into two-Group A - HPV positive and Group B - HPV negative. Pretreatment investigations done by the treating physician included complete blood count, renal function tests, liver function tests, CT Neck (from skull base to upper mediastinum). The results of these tests were recorded. Baseline performance status according to ECOG score were noted for each patient.

The standard treatment followed by majority of the physicians for oropharyngeal squamous cell carcinoma stage III and IV was induction chemotherapy with TPF 3 cycles followed by concurrent chemoradiation. Chemotherapy schedule includes Docetaxel-75 mg/m $\mathrm{m}^{2}$ on D1, Cisplatin- $60 \mathrm{mg} / \mathrm{m}^{2}$ on D1, 5-Fluorouracil-750 $\mathrm{mg} / \mathrm{m}^{2}$ on D1 and D2 every 3 weekly and premedication to avoid allergic reactions. Concurrent chemoradiation is cisplatin $40 \mathrm{mg} / \mathrm{m}^{2}$ weekly along with radiation 66 Gy as 33 fractions. Post treatment CT scan are taken after 3 months, 6 months and 1 year. Post treatment clinical examination including ENT evaluation was done 3 monthly for 1 st year and then 4 monthly thereafter. Treatment response was assessed using the RECIST criteria and entered into excel sheet. The treatment toxicity will be assessed in both groups using CTC version 4 . 


\section{Data Analysis}

Data analysis was done with the help of Excel 2010 and SPSS 16 statistical software. Student $\mathrm{T}$ test for continuous variables, Chi square test for categorical variables and Kaplan-Meier and log rank test for survival analysis.

\section{Results}

In this prospective observational study, we have enrolled a total of 65 pathologically proved squamous cell carcinoma of oropharynx who satisfied the selection criteria. Among this, 17 patients were HPV positive and 48 patients were HPV negative.

1) Baseline characteristics of the patients in this study are depicted in Table 1.

2) Clinical presentation

3) Table 2 Symptoms of the patient at presentation (Table 2).

4) Tumour characteristics (Table 3 ).

Table 1. Baseline characteristics of the cases in this study.

\begin{tabular}{|c|c|c|}
\hline Variable & HPV positive (Arm A) & HPV negative (Arm B) \\
\hline Mean age (yrs) & $51.18+/-9.1$ & $59.73+/-7.0$ \\
\hline Median age (yrs) & 48 & 59 \\
\hline \multicolumn{3}{|l|}{ Sex } \\
\hline Male & $58.8 \%$ & $68.7 \%$ \\
\hline Female & $41.2 \%$ & $31.3 \%$ \\
\hline Tobacco use present & $58.8 \%$ & $72.9 \%$ \\
\hline Alcohol use present & $58.8 \%$ & $68.8 \%$ \\
\hline Diabetes Mellitus & $29 \%$ & $25 \%$ \\
\hline Hypertension & $41 \%$ & $42 \%$ \\
\hline Both & $12 \%$ & $12 \%$ \\
\hline \multicolumn{3}{|l|}{ ECOG PS } \\
\hline 0 & $47 \%$ & $50 \%$ \\
\hline 1 & $35 \%$ & $33 \%$ \\
\hline 2 & $18 \%$ & $17 \%$ \\
\hline \multicolumn{3}{|l|}{ Socio Economic status } \\
\hline Below poverty line & $88 \%$ & $83 \%$ \\
\hline Above poverty line & $12 \%$ & $17 \%$ \\
\hline
\end{tabular}


Table 2. Clinical presentation.

\begin{tabular}{cccc}
\hline Symptoms & HPV Positive & HPV Negative & Total \\
& N (\%) & N (\%) & $34(52 \%)$ \\
\hline Dysphagia & $9(53 \%)$ & $25(52 \%)$ & $14(22 \%)$ \\
Swelling in neck & $4(25 \%)$ & $10(21 \%)$ & $8(12)$ \\
Swelling in oral Cavity & $2(12 \%)$ & $6(13 \%)$ & $5(8 \%)$ \\
Hoarseness & $1(5 \%)$ & $4(8 \%)$ & $4(6 \%)$ \\
Others & $1(5 \%)$ & $3(6 \%)$ & $65(100 \%)$ \\
Total & $17(100 \%)$ & $48(100 \%)$ &
\end{tabular}

Table 3. Tumour characteristics of the study population.

\begin{tabular}{|c|c|c|}
\hline Variable & $\mathrm{HPV}+(\mathrm{Arm} \mathrm{A})$ & $\mathrm{HPV}-(\mathrm{Arm} \mathrm{B})$ \\
\hline \multicolumn{3}{|l|}{ Tumour $(\mathrm{T})$} \\
\hline $\mathrm{T} 1$ & $23.5 \%$ & $12.5 \%$ \\
\hline $\mathrm{T} 2$ & $35.3 \%$ & $25 \%$ \\
\hline $\mathrm{T} 3$ & $29.4 \%$ & $41.7 \%$ \\
\hline $\mathrm{T} 4$ & $11.7 \%$ & $20.8 \%$ \\
\hline \multicolumn{3}{|l|}{ Node $(\mathrm{N})$} \\
\hline No & $5.9 \%$ & $18.8 \%$ \\
\hline $\mathrm{N} 1$ & $17.6 \%$ & $43.8 \%$ \\
\hline $\mathrm{N} 2$ & $23.5 \%$ & $22.9 \%$ \\
\hline N3 & $52.9 \%$ & $14.6 \%$ \\
\hline \multicolumn{3}{|l|}{ Composite stage } \\
\hline Stage III & $29.4 \%$ & $56.3 \%$ \\
\hline Stage IV & $70.6 \%$ & 43.8 \\
\hline \multicolumn{3}{|l|}{ Sub sites } \\
\hline Tonsil & $52.9 \%$ & $45.8 \%$ \\
\hline BOT & $29.4 \%$ & $25.0 \%$ \\
\hline Palate & $11.8 \%$ & $16.7 \%$ \\
\hline Vallecula & $5.9 \%$ & $12.5 \%$ \\
\hline \multicolumn{3}{|l|}{ Pathological subtypes } \\
\hline Well differentiated SCC & $11.8 \%$ & $14.6 \%$ \\
\hline Moderately differentiated SCC & $47.1 \%$ & $64.6 \%$ \\
\hline Poorly differentiated SCC & $23.5 \%$ & $18.8 \%$ \\
\hline Basaloid differentiation SCC & $11.8 \%$ & 0 \\
\hline Unknown differentiation SCC & $5.9 \%$ & $2.1 \%$ \\
\hline
\end{tabular}


5) HPV positivity-pattern of staining of P16 (Table 4).

6) Treatment response

The response to the planned treatment as per RECIST criteria is shown in $\mathrm{Ta}$ ble 5 .

7) Time to maximum response-Nearly $90 \%$ of the responders in both the arms showed the response to treatment in less than 6 month period (Table 6).

8) Toxicities-The major toxicities are enlisted as a table below (Table 7).

9) Recurrence pattern-The first failure pattern of the study population during the study period is given in Table 8.

Table 4. HPV staining pattern.

\begin{tabular}{cc}
\hline HPV p16 staining & N (out of 17 positives) \\
\hline $70 \%-79 \%$ & 2 \\
$80 \%-89 \%$ & 13 \\
$90 \%-99 \%$ & 2 \\
\hline
\end{tabular}

Table 5. Treatment response evaluation.

\begin{tabular}{ccc}
\hline Variable & HPV + & HPV - \\
\hline Complete response (CR) & $35.3 \%$ & $18.8 \%$ \\
Partial response (PR) & $47.1 \%$ & $33.3 \%$ \\
Stable disease (SD) & $5.9 \%$ & $27.1 \%$ \\
Progressive disease(PD) & $11.8 \%$ & $20.8 \%$ \\
\hline Treatment Response summary & & \\
Objective response (CR+PR) & $82.4 \%$ & $52.1 \%$ \\
No objective response & $17.6 \% \%$ & $47.9 \%$ \\
P value & & 0.029 \\
\hline
\end{tabular}

Table 6. Time to maximum response.

\begin{tabular}{cccc}
\hline & & HPV Positive & HPV Negative \\
& Objective response & $\mathrm{N}=14$ (responders) & $\mathrm{N}=25$ (responders) \\
& & $\mathrm{N}(\%)$ & $11(44 \%)$ \\
\hline 1 & $<3$ months & $7(50 \%)$ & $11(44 \%)$ \\
3 & $3-6$ months & $6(43 \%)$ & $3(12 \%)$ \\
\hline
\end{tabular}


Table 7. Major treatment toxicities.

\begin{tabular}{|c|c|c|}
\hline Toxicity & $\mathrm{HPV}+$ & $\mathrm{HPV}-$ \\
\hline \multicolumn{3}{|l|}{ Mucositis } \\
\hline Grade 0 & $5.9 \%$ & $6.3 \%$ \\
\hline Grade 1 & $11.8 \%$ & $12.5 \%$ \\
\hline Grade 2 & $41.2 \%$ & $41.7 \%$ \\
\hline Grade 3 & $35.3 \%$ & $33.3 \%$ \\
\hline Grade 4 & $5.9 \%$ & $6.3 \%$ \\
\hline \multicolumn{3}{|l|}{ Xerostomia } \\
\hline Grade 1 & $11.8 \%$ & $10.4 \%$ \\
\hline Grade 2 & $52.9 \%$ & $56.3 \%$ \\
\hline Grade 3 & $29.4 \%$ & $25.0 \%$ \\
\hline Grade 4 & $5.9 \%$ & $8.3 \%$ \\
\hline \multicolumn{3}{|l|}{ Dermatitis } \\
\hline Grade 0 & $5.9 \%$ & $6.3 \%$ \\
\hline Grade 1 & $11.8 \%$ & $12.5 \%$ \\
\hline Grade 2 & $41.2 \%$ & $41.7 \%$ \\
\hline Grade 3 & $35.3 \%$ & $33.3 \%$ \\
\hline Grade 4 & $5.9 \%$ & $6.3 \%$ \\
\hline
\end{tabular}

Table 8. Recurrence pattern.

\begin{tabular}{ccc}
\hline Failure pattern & HPV + & HPV - \\
\hline Loco-regional failure & $23.5 \%$ & $43.75 \%$ \\
Systemic failure & $5.9 \%$ & $6.25 \%$ \\
Total failure & $29.4 \%$ & $50 \%$ \\
\hline
\end{tabular}

\section{0) Progression free survival}

1 year progression free survival rate in HPV positive arm is $76.5 \%$ while in HPV negative arm it is $52.1 \%(\mathrm{p}$ value $=0.08)($ Figure 1$)$.

11) Overall survival

1 year overall survival in HPV positive arm is $82.4 \%$ while in HPV negative arm it is $70.8 \%$ (p value-0.353) (Figure 2). 


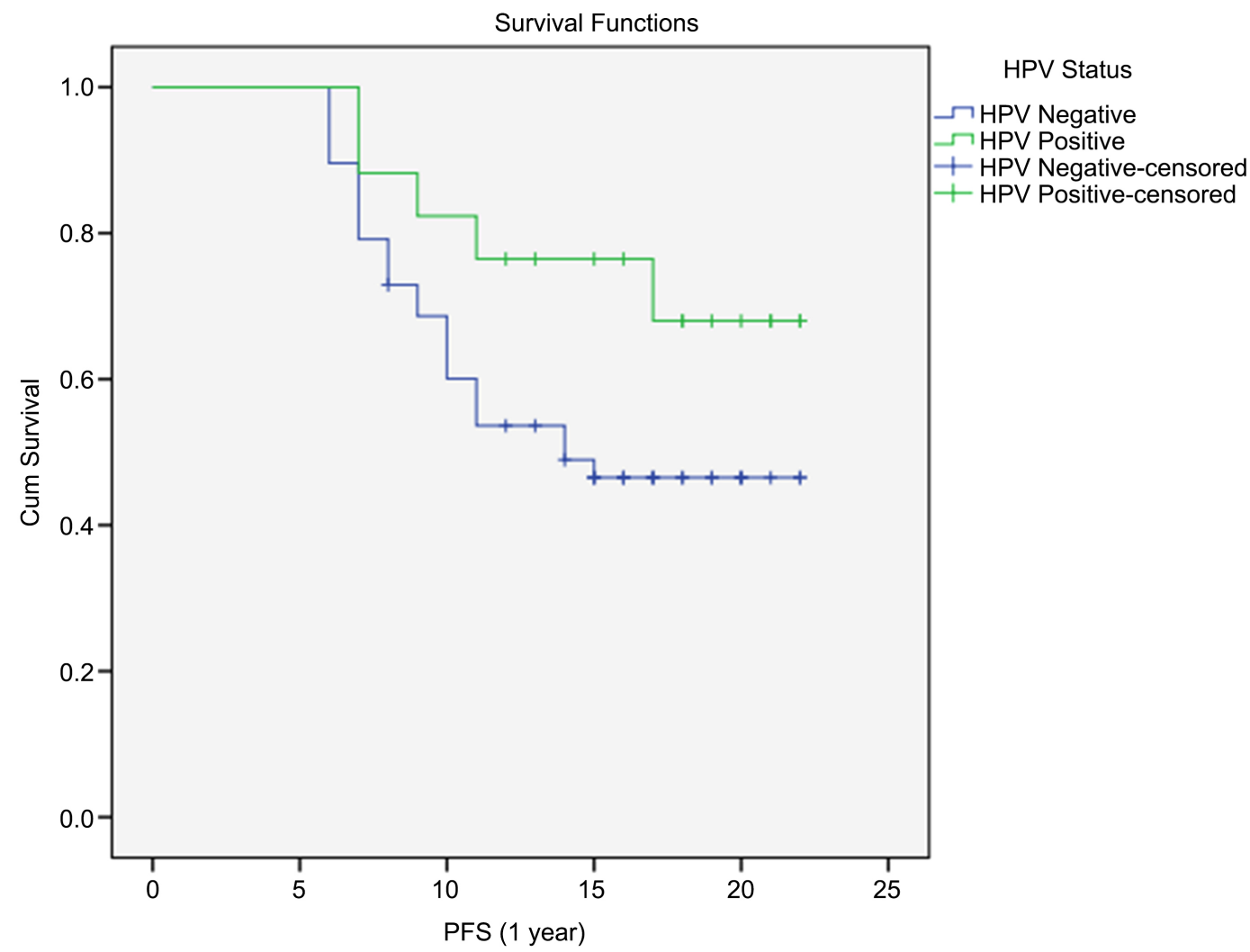

Figure 1. Kaplan Meir curve of progression free survival.

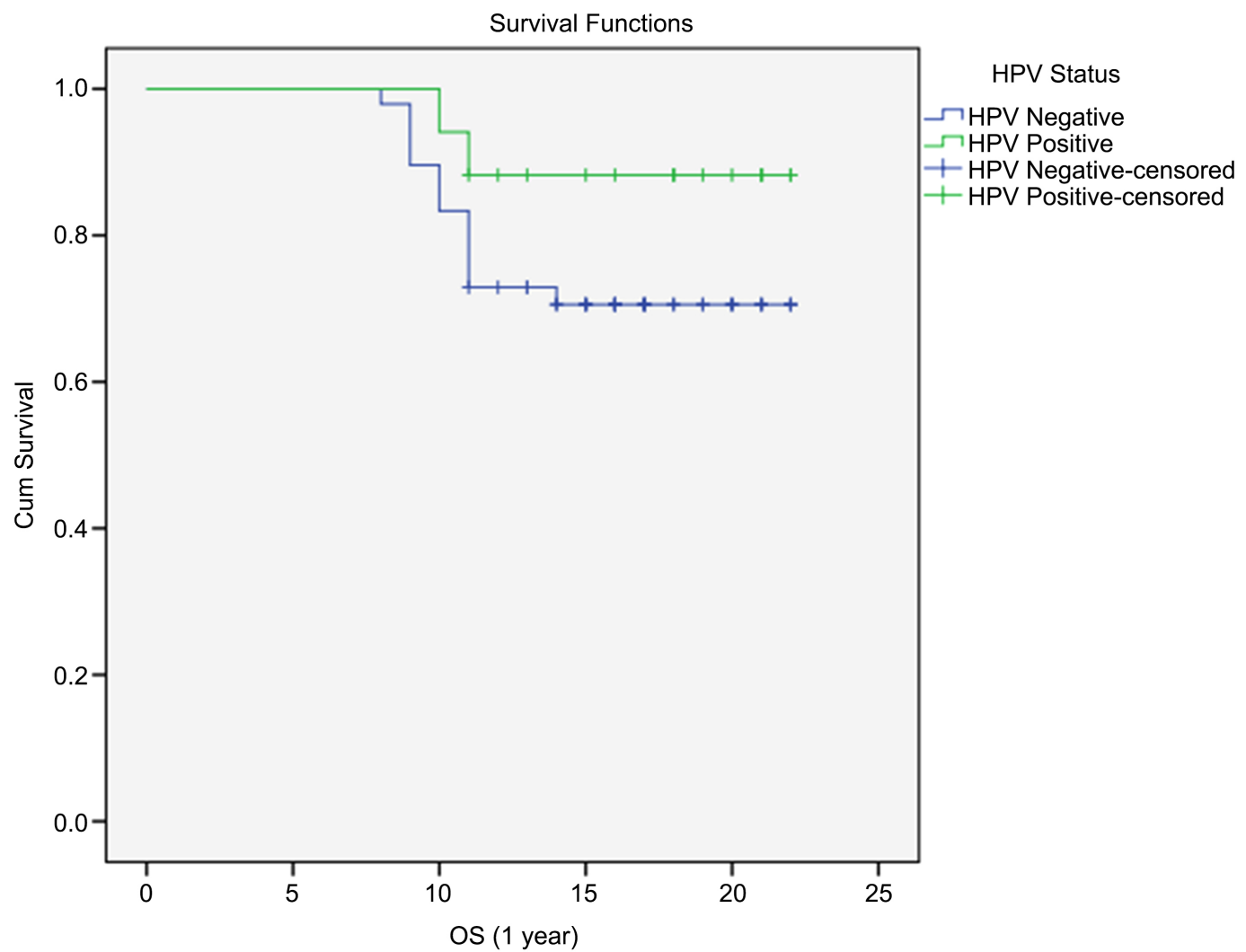

Figure 2. Kaplan Meir curve of overall survival. 


\section{Discussion}

The median age in HPV positive arm was 48 years while in HPV negative arm it was 59 years. This is similar to the findings of the study by Chaturvedi et al. in which HPV positive patients tend to be 5 to 10 years younger than HPV negative patients [6]. The male: female ratio in HPV positive arm is less which is correlated by the study by Hemminki et al. in which there is an increased risk of HPV associated OPSCC in female patients with a history of HPV associated anogenital cancers [7]. In HPV positive arm only $58.8 \%$ were tobacco users while in HPV negative it was $72.9 \%$. In HPV positive arm $58 \%$ had the habit of consuming alcohol while in HPV negative arm $68 \%$ were alcohol users. These results correlated with the findings of the study by D'Souza et al. in which HPV positive patients have less exposure to tobacco and alcohol [8].

With respect to the Tumour $(\mathrm{T})$ status, in HPV positive arm, 59\% patients had early $\mathrm{T}$ stage $(\mathrm{T} 1+\mathrm{T} 2)$ and $41 \%$ were of advanced $\mathrm{T}$ stage $(\mathrm{T} 3+\mathrm{T} 4)$. In contrast, HPV negative arm had only less proportion of early T stage (37.5\%) and a higher proportion of advanced $\mathrm{T}$ stage (67.5\%). This finding is again similar to the observations of the study by Huang et al., which have shown that HPV positive tumours are more likely to present with early T stage [9]. In HPV positive arm, 53.9\% patients presented with N3 nodal disease while in HPV negative arm $14.6 \%$ presented with N3 disease. A study by Goldenberg et al in has shown that HPV positive tumours present with higher $\mathrm{N}$ stage [10].

In both arms the most common histology was moderately differentiated SCC. In HPV positive patients poorly differentiated histopathology was seen in $23.5 \%$ of patients and basaloid SCC in $11.8 \%$ while in HPV negative patients, poorly differentiated was only $18.8 \%$ and no basaloid SCC. These findings show similar pattern as in the study by Gillison et al. [11], which showed that HPV positive tumours tend to be poorly differentiated and basaloid squamous cell carcinomas.

The incidence of HPV in the cohort is $26 \%$. A study by V Murthy et al. shows an incidence of HPV oropharyngeal cancer in India varies from $20 \%$ to $38 \%$ [12].

In HPV positive arm, the objective treatment response rate is $82 \%$ while in HPV negative arm the response rate is $52 \%$. There was statistically significant difference between two groups in case of treatment response ( $p$ value-0.029). Study by Fakhry et al. the treatment response in HPV positive arm was $84 \%$ and in HPV negative arm was 57\% [13] [14].

In HPV positive arm the 1 year progression free survival is $76.5 \%$ while in HPV negative arm the 1 year progression free survival is $52.1 \%$. But the difference between thetwo groups was not statistically significant ( $p$ value- 0.08 ). This may be due to the small sample size of the study.

In HPV positive arm the 1 year overall survival is $82.4 \%$ while in HPV negative arm the 1 year overall survival is $70.8 \%$. But the difference between the two arms was not statistically significant ( $\mathrm{p}$ value-0.353). Nguyen-Tan et al. [15] study showed improved PFS and OS in HPV positive OPSCC. In HPV positive 
arm only $30 \%$ of patients have recurrences while in HPV negative arm $50 \%$ of patients have recurrences. These results correlated with the study by Posner et al. [16].

The treatment related toxicities were almost similar in both arms. The percentage of grade 3 and 4 mucositis in HPV positive arm is 35.3\% and in HPV negative arm was $31.2 \%$.Grade 3 and 4 dermatitis in HPV positive arm is $41.2 \%$ and in HPV negative arm is $39.6 \%$. The results of these toxicities correlated with the study by Kanotra et al. [17].

\section{Conclusion}

The prevalence of HPV in the present study is $26 \%$ and the highest prevalence is noted in the tonsillar subsite of oropharynx. In this prospective observational study, we observed that there is a statistically significant difference in treatment response in HPV positive patients compared to HPV negative patients. Though there was no statistically significant difference between two arms in case of progression free survival and overall survival, there was a trend of better PFS and OS towards HPV positive patients. The limitation of our study is that due to small sample size a sub group analysis cannot be made based on the different characteristics. In view of better prognosis of HPV positive patients, several investigators are trying for de-escalation of treatment for HPV positive patients. HPV status in future will definitely play role in decision of treatment planning in oropharyngeal squamous cell carcinoma patients.

\section{Conflicts of Interest}

The authors declare no conflicts of interest regarding the publication of this paper.

\section{References}

[1] Mishra, A., et al. (2014) Head and Neck Cancer: Global Burden and Regional Trends in India. Asian Pacific Journal of Cancer Prevention, 15, 537-550. https://doi.org/10.7314/APJCP.2014.15.2.537

[2] Gillison, M.L., Koch, W. M., Capsone, R.B., et al. (2000) Evidence for a Causal Association between Human Papilloma Virus and a Subset of Head and Neck Cancers. Journal of the National Cancer Institute, 92, 709-720. https://doi.org/10.1093/jnci/92.9.709

[3] Torre, L.A., Bray, F., Seigel, R.L., et al. (2015) Global Cancer Statistics. CA: A Cancer Journal for Clinicians, 65, 87-108. https://doi.org/10.3322/caac.21262

[4] Chaturvedi, A.K., Engels, E.A., Pfeiffer, R.M., et al. (2011) Human Papilloma Virus and Rising Oropharyngeal Cancer Incidence in the United States. Journal of Clinical Oncology, 29, 4294-4301. https://doi.org/10.1200/JCO.2011.36.4596

[5] Nandakumar, A. (2001) National Cancer Registry Programme. Consolidated Report of the Population Based Cancer Registries: 1990-96. ICMR, Bangalore.

[6] Chaturvedi, A.K., Engels, E.A., Anderson, W.F., et al. (2008) Incidence Trends for Human Papilloma Virus Related and Unrelated Oral Squamous Cell Carcinomas in the United States. Journal of Clinical Oncology, 26, 612-619. 
https://doi.org/10.1200/JCO.2007.14.1713

[7] Hemminki, K., Dong, C. and Frisch, M. (2000) Tonsillar and Other Upper Aerodigeative Tract Cancers among Cervical Cancer Patients and Their Husbands. European Journal of Cancer, 9, 433-437. https://doi.org/10.1097/00008469-200012000-00010

[8] D’Souza, Kreimer, A.R., Clifford, G.M., et al. (2007) Case Control Study of Human Papillomavirus and Oropharyngeal Cancer. The New England Journal of Medicine, 356, 1944-1956. https://doi.org/10.1056/NEJMoa065497

[9] Huang, S.H., Peres-Ordonez, B., Liu, F.F., et al. (2012) A Typical Clinical Behaviour of p16-Confirmed HPV Related Oropharyngeal Squamous Cell Carcinoma Treated with Radical Radiotherapy. International Journal of Radiation Oncology Biology Physics, 82, 276-283. https://doi.org/10.1016/j.ijrobp.2010.08.031

[10] Goldenberg, D., Begum, S., Westra, W.H., et al. (2008) Cystic Lymph Node Metastasis in Patients with Head and Neck-Cancer: An HPV-Associated Phenomenon. Head Neck, 30, 898-903. https://doi.org/10.1002/hed.20796

[11] Gillison, M.L., et al. (2004) Human Papilloma Virus Associated Head and Neck Cancer Is a Distinct Epidemiologic, Clinical, and Molecular Entity. Seminars in Oncology, 31, 744-754. https://doi.org/10.1053/j.seminoncol.2004.09.011

[12] Murthy, V., et al. (2016) Human Papillomavirus/p16 Positive Head and Neck Cancer in India: Prevalence, Clinical Impact and Influence of Tobacco Use. Indian Journal of Cancer, 53, 387-393.

[13] Fakhry, C., et al. (2008) Improved Survival of Patients with Human Papillomavirus Positive Head and Neck Squamous Cell Carcinoma in a Prospective Clinical Trial. Journal of the National Cancer Institute, 100, 261-269. https://doi.org/10.1093/jnci/djn011

[14] Ang, K.K., Harris, J. and Wheeler, R. (2010) Human Papillomavirus and Survival of Patients with Oropharyngeal Cancer. The New England Journal of Medicine, 363, 24-35. https://doi.org/10.1056/NEJMoa0912217

[15] Nguyen-Tan, P.F., et al. (2014) Randomised Phase III Trial to Test Accelerated Versus Standard Fractionation in Combination with Concurrent Cisplatin for Head and Neck Carcinomas in RTOG 0129 Trial: Long Term Report of Efficacy and Toxicity. Journal of Clinical Oncology, 32, 3858-3867. https://doi.org/10.1200/JCO.2014.55.3925

[16] Posner, M.R., et al. (2011) Survival and Human Papilloma Virus in Oropharynx Cancer in TAX 324: A Subset Analysis from an International Phase III Trial. Annals of Oncology, 22, 1071-1077. https://doi.org/10.1093/annonc/mdr006

[17] Kanotra, S.P., et al. (2011) Chemoradiation in Advanced Head and Neck Cancers: A Comparison of Two Radiosensitizers, Paclitaxel and Cisplatin. Indian Journal of Otolaryngology and Head \& Neck Surgery, 63, 229-236.

https://doi.org/10.1007/s12070-011-0263-1 